\title{
Upcoming Events in Pediatric Cardiology
}

๑) Springer Science+Business Media, LLC, part of Springer Nature 2020

April 25-28, 2020

American Association of Thoracic Surgery, Annual Meeting New York, New York, USA

https://www.aats.org

July $19-23,2020$

American Society of Echocardiography, Annual Scientific Sessions

Denver, Colorado, USA

https://www.asecho.org

September 8-11, 2020

Pediatric Interventional Cardiology Symposium-AICS

Boston, Massachusetts, USA

https://www.picsymposium.com/

October 3-6, 2020

American Academy of pediatrics National Conference San Diego, California, USA

https://www.aapexperience.org/
November 14-16, 2020

Scientific Session, American Heart Association

Dallas, Texas, USA

https://professional.heart.org

January 30-February 2, 2021

Annual Meeting of the Society of Thoracic Surgery

Austin, Texas, USA

https://www.sts.org

March 20-22, 2021

American College of Cardiology, Annual Scientific Session Atlanta, Georgia, USA

https://www.acc.org

Publisher's Note Springer Nature remains neutral with regard to jurisdictional claims in published maps and institutional affiliations. 